ARTIGO DE REVISÃO REVIEW ARTICLE

\section{Análise de Decisão Multicritérios (MCDA): uma revisão rápida sobre os critérios utilizados na Avaliação de Tecnologias em Saúde}

\author{
Multicriteria Decision Analysis: rapid review about the \\ criteria used in the Health Technology Assessment
}

Andrea Brígida de Souza', Marisa da Silva Santos², Monica Akissue de Camargo Teixeira Cintra ${ }^{3}$

DOI: $10.21115 /$ JBES.v10.n1.p64-74

\section{Palavras-chave:}

avaliação da tecnologia

biomédica, tomada de decisões, técnicas de apoio para a decisão

\begin{abstract}
RESUMO
Objetivo: Identificar os vários critérios utilizados por agências e órgãos governamentais internacionais na Avaliação de Tecnologias em Saúde (ATS). Métodos: Foi realizada uma revisão rápida com busca estruturada na base de dados Medline (via PubMed) e Lilacs complementada por busca na literatura cinzenta, sem restrição de idioma. Incluíram-se estudos da área de saúde que descreviam critérios e que utilizaram o método MCDA (Análise de Decisão Multicritérios) na ATS. Excluíram-se estudos que avaliaram apenas uma tecnologia específica ou contexto restrito. Os critérios foram agregados e sumarizados conforme opinião de especialistas em ATS. Resultados: Foram identificadas 3.746 publicações, sendo 27 selecionadas. Os critérios extraídos foram categorizados e seus respectivos atributos foram listados. Dez categorias foram apresentadas: benefício e risco da intervenção (4 critérios); impacto da doença (4 critérios); impacto econômico (5 critérios); contexto terapêutico da intervenção (4 critérios); qualidade e incerteza da evidência (4 critérios); implementação da intervenção (5 critérios); benefícios para a sociedade (5 critérios); benefícios para o sistema de saúde público (5 critérios); benefícios para a indústria (1 critério); e outros (3 critérios). Os atributos mais prevalentes foram: custo-efetividade; segurança e tolerabilidade; impacto orçamentário para o sistema/plano de saúde; gravidade da doença; equidade; efetividade; custo. Conclusões: Os órgãos decisores utilizam grande variedade de critérios para o MCDA, refletindo visões e valores diferentes entre as culturas. O estudo fornece informação para discussão no contexto brasileiro de uma lista mais ampla e possível padronização de critérios a serem utilizados na tomada de decisão pública ou privada sobre a incorporação de novas tecnologias.
\end{abstract}

\section{Keywords:}

technology assessment, biomedical, decision-making, decision support techniques

\begin{abstract}
Objective: To identify the various criteria used by international government agencies and bodies in Health Technology Assessment (HTA). Methods: A rapid review was conducted with a structured search in the Medline database (via PubMed) and Lilacs supplemented by a search in the gray literature, without a restriction of language. Inclusion criteria were studies in health context, describing the criteria and using the MCDA (Multicriteria Decision Analysis) method in HTA. We excluded studies that assessed only one specific technology or restricted context. The criteria were aggregated and summarized according to the opinion of HTA experts. Results: A total of 3,746 publications were identified, of which 27 were selected. The extracted criteria were categorized, and their respective

\footnotetext{
Recebido em: 13/11/2017. Aprovado para publicação em: 16/04/2018

1. Departamento de Gestão e Incorporação de Tecnologias em Saúde, Secretaria de Ciência, Tecnologia e Insumos Estratégicos, Ministério da Saúde, Brasília, DF, Brasil.

2. Núcleo de Avaliação de Tecnologias em Saúde, Departamento de Ensino e Pesquisa, Instituto Nacional de Cardiologia, Ministério da Saúde, Rio de Janeiro, RJ, Brasil.

3. Unidade de Pesquisa Clínica, Hospital das Clínicas da Faculdade de Medicina de Ribeirão Preto, Universidade de São Paulo, Ribeirão Preto, SP, Brasil.

Instituição onde o trabalho foi executado: Instituto Nacional de Cardiologia, Ministério da Saúde, Rio de Janeiro, RJ, Brasil. Fontes externas de financiamento: Não foram recebidos auxílios na forma de financiamento, equipamentos ou medicamentos. 0 estudo não foi apresentado em congressos científicos.

Conflitos de interesse: Não há potenciais conflitos de interesse.

Autora correspondente: Andrea Brígida de Souza. Esplanada dos Ministérios, Ministério da Saúde, Edifício Sede, bloco G, $8^{\circ}$ andar, sala 853. Departamento de Gestão e Incorporação de Tecnologias em Saúde (DGITS/SCTIE/MS). Brasília-DF, Brasil. CEP: 70058-900. Telefone: (61) 3315-3502.E-mail: brigida. andrea@gmail.com
} 
attributes were listed. Ten categories were presented: benefit and risk of the intervention (4 criteria); impact of disease (4 criteria); economic impact ( 5 criteria); therapeutic context of the intervention (4 criteria); quality and uncertainty of evidence (4 criteria); implementation of the intervention (5 criteria); benefits for society (5 criteria); benefits to the public health system (5 criteria); benefits for industry (1 criterion); and others (3 criteria). The most prevalent attributes were: cost-effectiveness; safety and tolerability; budget impact for the health system/plan; disease severity; equity; effectiveness; cost. Conclusions: Decision-makers use a wide variety of criteria for the MCDA, reflecting different views and values across cultures. The study provides information for a discussion in the Brazilian context of a broader list and a possible standardization of criteria to be used in public or private decision making on the incorporation of new technologies.

\section{Introdução}

Os gestores públicos precisam tomar importantes decisões sobre a alocação adequada dos recursos de saúde (Tony et al., 2011; Baltussen \& Niessen, 2006). Com o crescente desenvolvimento de novas tecnologias em saúde, o estabelecimento de critérios de priorização é de fundamental importância para que o sistema de saúde escolha as tecnologias em saúde que mais ofertem bem-estar social (Baltussen \& Niessen, 2006; Youngkong et al., 2012a; Youngkong, 2014). Esse tipo de julgamento inclui a possibilidade de uso de muitos critérios com importâncias diferenciadas a serem considerados (Baltussen \& Niessen, 2006).

O processo decisório demanda transparência, reprodutibilidade e responsabilidade para que seja considerado legítimo pelo público e pelas prestadoras de serviço (Baltussen \& Niessen, 2006; Dhalla \& Laupacis, 2008). Dessa forma, os múltiplos critérios envolvidos devem ser considerados simultaneamente e facilmente estarão em conflito uns com os outros, logo, precisam ser selecionados e valorados adequadamente. Há dificuldade em se organizar e sintetizar cada informação, portanto ter uma ferramenta que auxilie nessa tomada de decisão e permita que os gestores se sintam mais confortáveis e confiantes em suas decisões, minimizando o potencial arrependimento após uma deliberação. Assim, essa ferramenta torna-se uma forma de apoio e legitimidade à tomada de decisão (Belton \& Stewart, 2002).

O Multi-Criteria Decision Analysis (Análise de Decisão Multicritérios - MCDA) é um termo amplo ("umbrella term") utilizado para descrever um processo decisório, que auxilia uma tomada de decisão (Baltussen \& Niessen, 2006; Belton \& Stewart, 2002; Devlin \& Sussex, 2011; Diaby \& Goeree, 2014). O MCDA é um conjunto de métodos e abordagens (quantitativa e qualitativa) que busca ordenar os vários critérios explícitos, os quais possuem, cada um, uma importância relativa distinta, sendo, portanto, atribuídos pesos diferentes (Baltussen \& Niessen, 2006; Belton \& Stewart, 2002; Devlin \& Sussex, 2011). O MCDA permite abordar problemas complexos e gerenciá-los, dividindo o problema em critérios menores, facilitando a avaliação e o debate, e identificando mais facilmente as concordâncias e discordâncias (Dodgson et al., 2009).
O MCDA permite abordagem transparente e estruturada, que facilita a reprodutibilidade da tomada de decisão e deixa clara a importância relativa de diferentes critérios (Devlin \& Sussex, 2011; Thokala \& Duenas, 2012). Outra característica importante nessa análise é a de evitar a redundância dos termos, no qual os critérios devem ser cuidadosamente identificados para que não haja dupla contagem (Diaby \& Goeree, 2014). Além disso, uma análise multicritérios deve ser abrangente de forma a garantir que todos os aspectos importantes serão considerados na tomada de decisão, principalmente quando diferentes interessados (stakeholders) fazem o julgamento. Neste ponto possuir uma ampla lista de critérios previamente escolhidos com base nos valores dos decisores pode apoiar análises importantes do ponto de vista de pacientes como equidade e conforto, tornando, em teoria, a tomada de decisão mais justa.

O uso do MCDA na área de saúde é incipiente comparado às outras áreas como ecologia. A dificuldade em tomar uma decisão nesse campo e a necessidade de se justificar publicamente tal julgamento justifica o uso do MCDA (Devlin \& Sussex, 2011; Thokala \& Duenas, 2012).

No Brasil, a Comissão Nacional de Incorporação de Tecnologias no Sistema Único de Saúde - SUS (Conitec), criada por meio da Lei no 12.401, de 28 de abril de 2011 (Brasil, 2011a), e do Decreto no 7.646, de 21 de dezembro de 2011 (Brasil, 2011b), tem como objetivo assessorar o Ministério da Saúde nas atribuições relativas à incorporação, à exclusão ou à alteração de tecnologias em saúde pelo SUS, bem como na constituição ou na alteração de Protocolos Clínicos e Diretrizes Terapêuticas (PCDT). A legislação citada estabelece os critérios fundamentais que deverão estar presentes nessas avaliações, sendo estes: evidências científicas sobre eficácia, acurácia, efetividade e segurança do medicamento, produto ou procedimento em análise, assim como avaliação econômica comparativa dos benefícios e dos custos em relação às tecnologias já incorporadas no SUS e o impacto orçamentário da incorporação nesse sistema de saúde (Brasil, 2011a; Brasil, 2011b). Verifica-se, no entanto, que, apesar de não estarem explícitos na legislação, na prática vários outros critérios importantes são levados em consideração nesse processo de decisão, a depender do relator ou da opinião dos membros da Comissão. 
O objetivo desta revisão rápida foi identificar e descrever os critérios utilizados internacionalmente em tomadas de decisão em saúde utilizando quaisquer técnicas de MCDA.

\section{Métodos}

Foram incluídos estudos da área de saúde e que utilizaram o método MCDA na Avaliação de Tecnologias em Saúde. Os estudos deveriam incluir obrigatoriamente a descrição dos critérios considerados nas tomadas de decisão. Excluíram-se estudos que avaliaram apenas uma situação específica, por exemplo, uma doença, um patógeno, um equipamento médico ou um exame. A razão da exclusão foi a aplicação de critérios muito específicos relacionados ao contexto, sem validade externa.

A busca estruturada foi realizada na base de dados Medline (via PubMed) e Lilacs; incluídos estudos até 03/02/2017. Não se restringiu idioma ou tipo de estudo. A seguinte estratégia de pesquisa foi utilizada:

(("Decision Making"[Mesh] NOT shar*[TIAB]) OR ("Decision Support Techniques"[Mesh])) AND ("multiple criteria decision*"[tiab] OR ((multiple[tiab] AND criteria[tiab]) OR (multiple[tiab] AND decision*[tiab])) OR "multi-criteria decision*"[tiab] OR "MCDA"[tiab] OR "MCDM"[tiab])

Em adição às bases indexadas, foi realizada uma busca na literatura cinzenta a partir das seguintes bases: sítio eletrônico de busca (Google acadêmico), sítio eletrônico da Universidade de York (Centre for Reviews and Dissemination - CRD), referências bibliográficas dos artigos incluídos e em sítio eletrônico de congresso internacional: International Society for Pharmacoeconomics and Outcomes Research (ISPOR) e Health Technology Assessment international (HTAi). Além disso, foi realizada busca manual na lista das referências localizadas.

A seleção dos estudos foi realizada de acordo com os critérios de elegibilidade definidos previamente, por dois revisores independentes (ABS e MACTC) com base na análise de títulos, resumos e artigos completos. As discordâncias foram resolvidas por um terceiro revisor (MSS).

Os dados foram extraídos para um formulário padronizado predefinido. As informações incluíam autor e ano da publicação, desenho do estudo, aplicação do MCDA, tipo de tecnologia avaliada, avaliador, método de MCDA e quantidade de critérios.

As opções para o desenho do estudo foram revisão narrativa, revisão sistemática e estudo de caso. A aplicação do MCDA incluiu "investimento", "financiamento de pesquisa", "prescrição médica ou hospitalar", "registro" ou "não relatado". Os tipos de tecnologias avaliadas foram medicamentos, equipamentos e dispositivos médicos (devices), programas de saúde, pesquisas, várias intervenções em saúde ou não especificado. $O$ avaliador ou tomador de decisão poderiam ser gestores de sistema de saúde (público ou privado), profissionais de saúde, agências reguladoras, indústria farmacêutica, agência de fomento à pesquisa, pesquisadores ou diversos agentes de decisão. O tipo de modelo de MCDA foram denominados "Value measurement model", "Outranking model" ou "Goal programming". Todos os critérios citados, seus atributos (forma com que os critérios são mensurados) e suas respectivas categorias foram descritos.

\section{Resultados}

A busca inicial nas bases bibliográficas localizou 3.746 artigos (Figura 1). Foram selecionados para leitura completa 102, dos quais 79 foram excluídos: 9 por não serem da área de saúde; 24 por não serem MCDA; 10 por, apesar de serem MCDA, não descreveram critérios; 36 por, apesar de serem da saúde e MCDA, não descreverem critérios em ATS e serem voltados a situações específicas. Ao final, 27 artigos foram escolhidos para a seleção dos critérios. A busca manual realizada na literatura cinzenta localizou mais 3 artigos primários e 1 revisão narrativa da literatura.

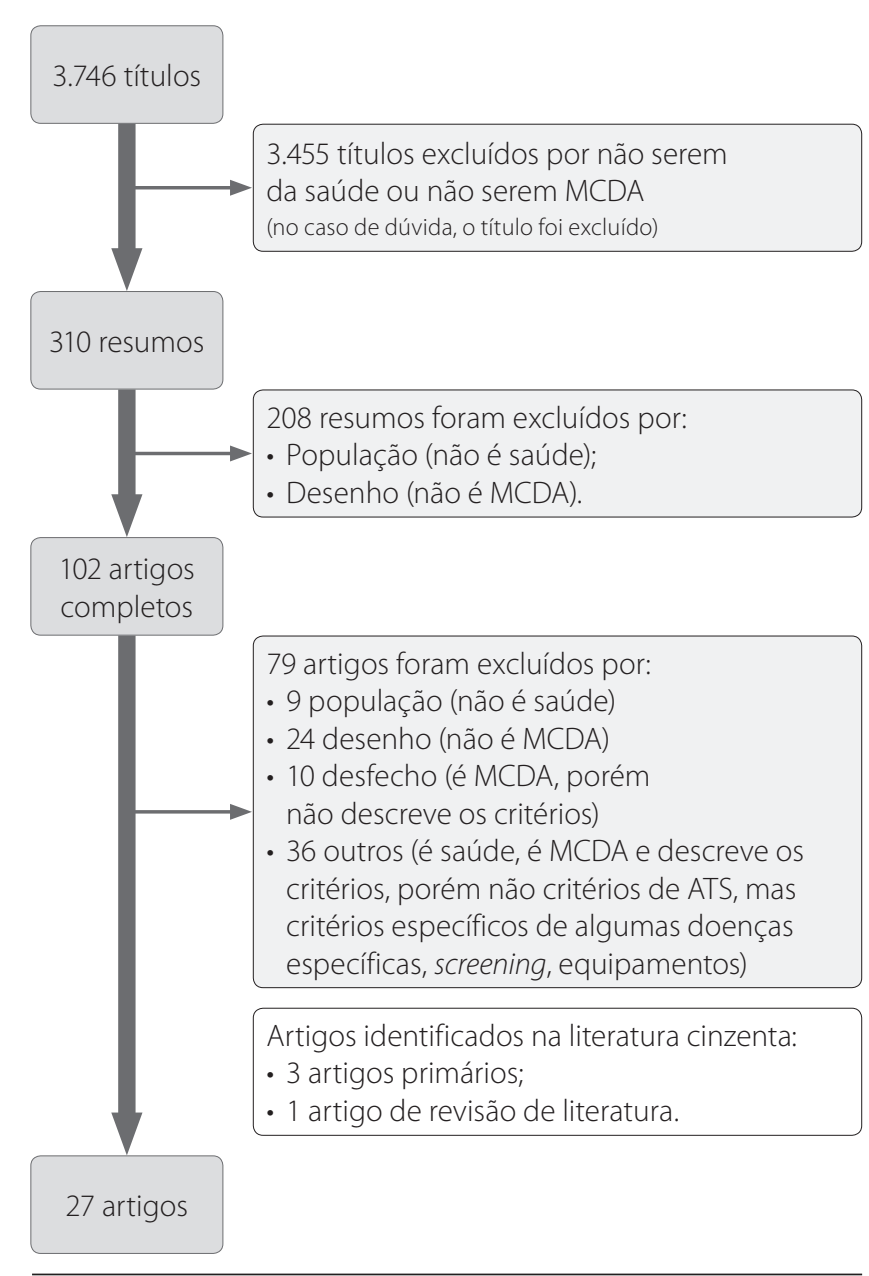

Figura 1. PRISMA de seleção dos artigos. 
Foram incluídos 27 artigos científicos. Na maioria dos estudos ( $n=19$ ), o MCDA era utilizado na saúde para decisões de investimento, ou seja, na priorização de intervenções para cobertura e reembolso pelo sistema de saúde do país, seja público ou privado (planos de saúde). Três estudos tratavam de prescrição de tecnologias em um hospital e um estudo utilizou o MCDA no financiamento de pesquisa.

A maioria dos artigos avaliaram medicamentos $(n=9)$ ou diversas tecnologias ao mesmo tempo $(n=12)$. Decisores políticos ou gestores foram os principais avaliadores $(n=13)$ e o tipo de modelo de MCDA mais utilizado foi a Value measurement model $(n=14)$.

Todos os critérios selecionados foram categorizados de acordo com seu conceito, sendo descritos seus respectivos atributos, ou seja, as medidas utilizadas para definir o critério (Tabela 1 e Figura 2).

O sistema de classificação apresenta 10 categorias: benefício e risco da intervenção (4 critérios); impacto da doença (4 critérios); impacto econômico (5 critérios); contexto terapêutico da intervenção (experiência prévia com a tecnologia)

Tabela 1. Principais critérios e atributos de cada categoria

\begin{tabular}{|c|c|c|}
\hline Categoria & Critérios & Atributos \\
\hline \multirow[t]{4}{*}{$\begin{array}{l}\text { Benefício e risco } \\
\text { da intervenção }\end{array}$} & Eficácia e efetividade & 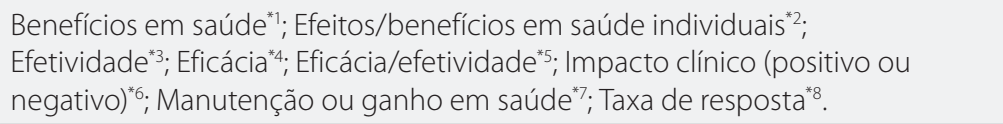 \\
\hline & Segurança & Eventos adversos ou efeitos colaterais ${ }^{*}$; Ris $\cos ^{* 10}$; Segurança e tolerabilidade ${ }^{* 11}$. \\
\hline & Impacto na sobrevida & $\begin{array}{l}\text { Benefício médio em anos de vida por paciente }{ }^{* 12} \\
\text { Prolongamento da sobrevida livre de doença }\end{array}$ \\
\hline & $\begin{array}{l}\text { Desfechos centrados } \\
\text { no paciente }\end{array}$ & $\begin{array}{l}\text { Conforto e conveniência do paciente }{ }^{* 14} ; \text { Impacto da qualidade de vida }{ }^{* 15} ; \\
\text { Melhoria do desfecho relatado pelo paciente }{ }^{* 16} ; \text { Melhoria dos resultados } \\
\text { relatados pelo paciente, conveniência e adesão*17; Número de QALY ganho } \\
\text { por paciente }{ }^{* 18} \text {; Preferência do paciente }{ }^{* 19} ; \text { Qualidade de vida }{ }^{* 20} \text {; Utilidade }{ }^{* 21} \text {. }\end{array}$ \\
\hline \multirow[t]{4}{*}{ Impacto da doença } & Gravidade da doença & Gravidade da doença ${ }^{* 22}$. \\
\hline & Carga da doença & Carga da doença ${ }^{* 23}$. \\
\hline & Epidemiologia & $\begin{array}{l}\text { Grupo-alvo da intervençãa**24; Número de potenciais beneficiários }{ }^{* 25} \text {; Prevalência } \\
\text { ou incidência da doença }{ }^{* 26} ; \text { Tamanho da população afetada/alvo }{ }^{* 27} \text {. }\end{array}$ \\
\hline & Prevenção & Prevenção*28. \\
\hline \multirow[t]{5}{*}{ Impacto econômico } & Custo-efetividade & $\begin{array}{l}\text { Avaliações econômicas }{ }^{* 29} \text {; Custo-efetividade }{ }^{* 30} \text {; } \\
\text { Custo e consequências }{ }^{* 13} \text {; Eficiência }{ }^{* 32} \text {. }\end{array}$ \\
\hline & Impacto orçamentário & $\begin{array}{l}\text { Capacidade para pagar (affordability) }{ }^{* 33} \text {; Impacto } \\
\text { orçamentário para o sistema/plano de saúde }{ }^{* 34} \text {. }\end{array}$ \\
\hline & $\begin{array}{l}\text { Custos/valores } \\
\text { do tratamento }\end{array}$ & $\begin{array}{l}\text { Custos }{ }^{* 35} \text {; Custo unitário*36; Maximização da utilidade/ } \\
\text { benefício por unidade monetária (Value for money) }{ }^{* 37} \text {. }\end{array}$ \\
\hline & $\begin{array}{l}\text { Impacto financeiro } \\
\text { amplo }\end{array}$ & Economia de custo $^{* 38}$; Impacto em outras despesas ${ }^{* 39}$. \\
\hline & Custo de oportunidade & Custos de oportunidade à população/sociedade*40. \\
\hline \multirow{4}{*}{$\begin{array}{l}\text { Contexto terapêutico da } \\
\text { intervenção (experiência } \\
\text { prévia com a tecnologia) }\end{array}$} & $\begin{array}{l}\text { Alternativas de } \\
\text { tratamento }\end{array}$ & $\begin{array}{l}\text { Alternativas de tratamento disponíveis } s^{* 41} \text {; Limitações das intervenções } \\
\text { comparativas/atuais (necessidades não atendidas) }{ }^{* 2} \text {. }\end{array}$ \\
\hline & Necessidade & Necessidade $^{* 43}$; Necessidade clínica ${ }^{* 44}$ \\
\hline & $\begin{array}{l}\text { Diretrizes clínicas } \\
\text { \& práticas }\end{array}$ & Diretrizes baseadas em evidências ${ }^{*}$; ; Diretrizes clínicas ${ }^{*}$. \\
\hline & Uso preexistente & Prescrição preexistente de medicamento ${ }^{*}$. \\
\hline \multirow{4}{*}{$\begin{array}{l}\text { Qualidade e incerteza } \\
\text { da evidência }\end{array}$} & Evidência disponível & Evidência científica*48; Tempo do uso da tecnologia*49. \\
\hline & Força da evidência & $\begin{array}{l}\text { Completude e consistência da evidência relatada } a^{* 50} \text {; Consistência }{ }^{* 51} \text {; Força da } \\
\text { evidência*52; Grau de certeza }{ }^{* 53} \text {; Qualidade da evidência }{ }^{*} \text {; } \text { Transparência*55. }\end{array}$ \\
\hline & Relevância da evidência & $\begin{array}{l}\text { Nível de generalização }{ }^{* 56} \text {; Relevância e validade da } \\
\text { evidência*57; Representatividade dos usuários }{ }^{* 58} \text {. }\end{array}$ \\
\hline & Relevância do desfecho & Escolha dos desfechos ${ }^{*} 59$ \\
\hline
\end{tabular}




\begin{tabular}{|c|c|c|}
\hline \multirow{5}{*}{$\begin{array}{l}\text { Implementação } \\
\text { da intervenção }\end{array}$} & Legislação & Implicações legais" 60 . \\
\hline & Habilidades & Complexidade técnica ${ }^{* 61}$; Disponibilidade de recursos humanos ${ }^{* 62}$. \\
\hline & $\begin{array}{l}\text { Requisitos } \\
\text { organizacionais e } \\
\text { implementação }\end{array}$ & $\begin{array}{l}\text { Aceitação }{ }^{* 63} \text {; Engajamento }{ }^{* 64} \text {; Implementação*65; Meio ambiente }{ }^{* 66} \text {; Processo }{ }^{* 67} \text {; } \\
\text { Tipo de serviço médico* }{ }^{* 68} \text {; Viabilidade organizacional }{ }^{* 69} \text {; Viabilidade de adoção }{ }^{* 70} \text {. }\end{array}$ \\
\hline & $\begin{array}{l}\text { Integração e } \\
\text { sistema eficiente }\end{array}$ & 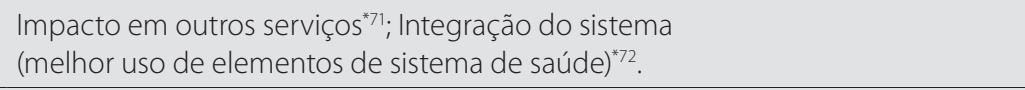 \\
\hline & $\begin{array}{l}\text { Características } \\
\text { da tecnologia }\end{array}$ & $\begin{array}{l}\text { Autonomia/interdependência da tecnologia*73; Características } \\
\text { da tecnologia*74; Sustentabilidade da tecnologia }{ }^{* 75} \text {. }\end{array}$ \\
\hline \multirow{5}{*}{$\begin{array}{l}\text { Benefícios para } \\
\text { a sociedade }\end{array}$} & Prioridade da população & Prioridades ${ }^{* 76}$; Prioridades nacionais e locais ${ }^{* 77}$. \\
\hline & Acesso & Acesso ${ }^{* 8}$; Equidade de acesso*79. \\
\hline & $\begin{array}{l}\text { Impacto social e } \\
\text { vulnerabilidade }\end{array}$ & 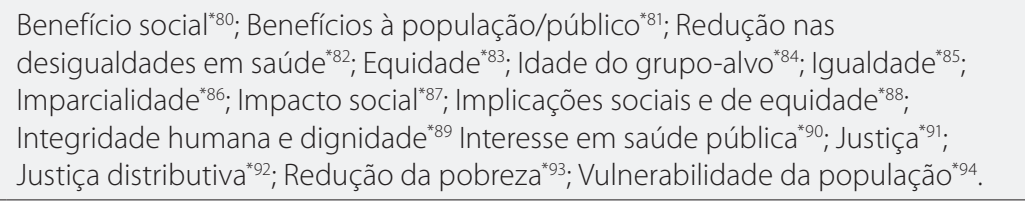 \\
\hline & Solidariedade & Confiança recíproca*95; Solidariedade ${ }^{* 96}$. \\
\hline & $\begin{array}{l}\text { Aspectos morais } \\
\text { e éticos }\end{array}$ & $\begin{array}{l}\text { Beneficência/não maleficência }{ }^{* 97} \text {; Consequência moral da ATS }{ }^{* 98} ; \\
\text { Consistência com valores da sociedade }{ }^{* 99} \text {; Obrigação moral em } \\
\text { implementar a tecnologia }{ }^{* 100} ; \text { Valores éticos }{ }^{* 101} ; \text { Valores e } \text { crenças }^{* 102} \text {. }\end{array}$ \\
\hline \multirow[t]{5}{*}{$\begin{array}{l}\text { Benefícios para o sistema } \\
\text { de saúde público }\end{array}$} & $\begin{array}{l}\text { Missão e mandato do } \\
\text { sistema de saúde }\end{array}$ & $\begin{array}{l}\text { Ajuste estratégico }{ }^{* 103} \text {; Consenso social e presente }{ }^{* 104} \text {; Consenso sobre } \\
\text { financiamento público de uma terapia }{ }^{* 105} ; \text { Metas da saúde }{ }^{* 106} \text {. }\end{array}$ \\
\hline & Incentivos & $\begin{array}{l}\text { Apoio organizacional }{ }^{* 107} ; \text { Envolvimento do financiador }{ }^{* 108} \\
\text { Incentivos financeiros }{ }^{* 109} ; \text { Incentivos por adesão*110. }\end{array}$ \\
\hline & Inovação & Inovação*111. \\
\hline & Parceria e liderança & Compromisso acadêmico: pesquisa e educação ${ }^{* 112} ;$ Parcerias e rede de trabalho ${ }^{* 113}$ \\
\hline & $\begin{array}{l}\text { Envolvimento } \\
\text { do cidadão }\end{array}$ & $\begin{array}{l}\text { Alfabetização em saúde/empoderamento }{ }^{* 114} ; \text { Cidadania*115; }^{*} \\
\text { Impacto na educação em saúde }{ }^{* 116 ;} \text {; Propriedade }{ }^{* 117} \text {. }\end{array}$ \\
\hline $\begin{array}{l}\text { Benefícios para } \\
\text { a indústria }\end{array}$ & $\begin{array}{l}\text { Interesses e pressões } \\
\text { dos interessados } \\
\text { (stakeholders) }\end{array}$ & $\begin{array}{l}\text { Militância (advocacy)*118; Pressão do médico/grupos de pacientes e } \\
\text { decisões anteriores }{ }^{* 119} \text {; Pressão dos interessados (stakeholders) }{ }^{* 120} \text {. }\end{array}$ \\
\hline \multirow[t]{3}{*}{ Outros } & Aspectos políticos & Contexto político*121; Pressão governamental ${ }^{* 122}$. \\
\hline & Aspectos históricos & Componentes históricos ${ }^{* 123}$. \\
\hline & Aspectos culturais & Convicções religiosas e culturais ${ }^{* 124}$. \\
\hline
\end{tabular}

" Diaby \& Goeree, 2014; Dionne et al., 2009; Gibson et al., 2005; Golan \& Hansen, 2012; Kapiriri et al., 2009; Kolasa et al., 2016; Mühlbacher \& Kaczynski, 2015; Youngkong et al., 2009. 2Baltussen et al., 2007; Defechereux et al., 2012; Duthie et al., 1999; Golan \& Hansen, 2010; Venhorst et al., 2014; Youngkong et al., 2009. "3 Dionne et al., 2015; Dolan, 2010; Duthie et al., 1999; Le Gales \& Moatti, 1990; Gibson et al., 2006; Gurtner, 2014; Hilgerink et al., 2011; Johnson et al., 2009; Lasry et al., 2011; Mullen, 2004; Ritrovato et al., 2015; Tannahill, 2008; Wilson et al., 2006; Wilson et al., 2007; Youngkong et al., 2012a; Youngkong et al., 2012b. ${ }^{{ }^{* 4}}$ de Greef-van der Sandt et al., 2016; Vuorenkoski et al., 2008; Youngkong et al., 2009. ${ }^{\text {*5 }}$ Diaby \& Goeree , 2014; Goetghebeur et al., 2008; Goetghebeur et al., 2010; Goetghebeur et al., 2012; Golan \& Hansen, 2010; Hailey, 2009; Menon et al., 2010; Miot et al., 2012; Mullen, 2004; Tony et al., 2011; University of York, 2002; Wilson et al., 2007. "6 Golan \& Hansen, 2010; Johnson et al., 2009; Martin et al., 2001; Meagher, 2010; Noorani et al., 2007; Simoens, 2014; Vuorenkoski et al., 2008. 7 Bots \& Hulshof, 2000; Koopmanschap etal. 2010; Mullen, 2004. " ${ }^{8}$ Broekhuizen et al. 2015; Mitton et al. 2006. "9 Broekhuizen et al. 2015; Dionne et al. 2015; Dolan, 2010; Duthie et al., 1999; Johnson et al., 2009; Lehoux \& Williams-Jones, 2007; Lopert, 2009; Mühlbacher \& Kaczynski, 2015; Vuorenkoski et al., 2008. "10 Hailey, $2009 ;$ Johnson et al., $2009 ;$ Martin et al., 2001. "II Adunlin et al., 2015; Andreae et al., 2009; de Greef-van der Sandt et al., 2016; Diaby \& Goeree, 2014; Dionne et al., 2009; Goetghebeur et al., 2008; Goetghebeur et al., 2010; Goetghebeur et al., 2012; Golan \& Hansen, 2010; Gurtner, 2014; Hailey, 2009; Hilgerink et al., 2011; Johnson et al., 2009; Miot et al., 2012; Ritrovato et al., 2015; Sloane et al., 2003; Tannahill, 2008; Tony et al., 2011; Tromp \& Baltussen, 2012; Venhorst et al., 2014. "12 Duthie et al., 1999; Youngkong et al., 2009. " ${ }^{13}$ Martin et al., 2001; Mühlbacher \& Kaczynski, 2015. " 14 Hilgerink et al., 2011; Lehoux \& Williams-Jones, 2007; Martin et al., 2001." ${ }^{15}$ Johnson et al., 2009; Mitton et al., 2006. " ${ }^{16}$ Goetghebeur et al., 2010; Miot et al., 2012. "17 Adunlin et al., 2015; Goetghebeur et al., 2008; Goetghebeur et al., 2012; Mühlbacher \& Kaczynski, 2015; Thokala \& Duenas, 2012; Tony et al., 2011. "18 Irving et al., 2010; Koopmanschap et al., 2010. * "19 Hofmann, 2005; Lehoux \& Williams-Jones, 2007; Menon et al., 2010; Tannahill, 2008. "20 Golan \& Hansen, 2010; Hummel et al., 2012; Martin et al., 2001; Mühlbacher \& Kaczynski, 2015; Mullen, 2004; Wilson et al., 2006; Wilson et al., 2009. "21 Goetghebeur et al., 2010; Gurtner, 2014; Menon et al, 2010; Tannahill, 2008. "22 Andreae et al., 2009; Baltussen et al., 2006; Baltussen et al., 2007; Defechereux et al., 2012; Diaby \& Goeree, 2014; Dionne et al., 2015; Goetghebeur et al., 2008; Goetghebeur et al., 2010; Goetghebeur et al., 2012; Golan \& Hansen, 2010; Jehu-Appiah et al., 2008; Kolasa et al., 2016; Koopmanschap et

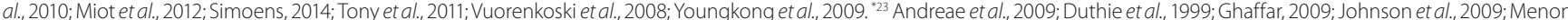
et al., 2010; Noorani et al., 2007; Tromp \& Baltussen, 2012; Wilson et al., 2007; Youngkong et al., 2009; Youngkong, 2014. ${ }^{24}$ Marsh et al., 2013; Youngkong et al., 2012 b. ${ }^{~}{ }^{25}$ Baltussen et al., 2007 ; Defechereux et al., 2012; Jehu-Appiah et al., 2008; Hofmann, 2005; Lehoux \& Williams-Jones, 2007." ${ }^{22}$ Andreae et al., 2009; Dionne et al., 2015; Johnson et al., 2009 ; Kirby et al., 2008; Menon et al. 2010; Mitton et al. 2006; Youngkong et al. 2009. '27 Goetghebeur et al. 2008; Goetghebeur et al., 2010; Goetghebeur et al. 2012; Golan \& Hansen, 2010; Miot et al., 2012; Tony et al., 2011; Tromp \& Baltussen, 2012; Youngkong et al., 2012a. "28 Dionne et al., 2015; Golan \& Hansen, 2010; Mullen, 2004; Wilson et al., $2006 .{ }^{* 29}$ Diaby \& Goeree, 2014; Ritrovato et al., 2015; University of York, 2002. ${ }^{30}$ Andreae et al., 2009; Baltussen et al., 2006; Baltussen et al., 2007; Defechereux et al., 2012; Diaby \& Goeree, 2014; Diaby \& Lachaine, 2011; Ghaffar, 2009; Gibson et al., 2006; Goetghebeur et al., 2008; Goetghebeur et al., 2010; Goetghebeur et al., 2012; Gurtner, 2014; Hailey, 2009; Jehu-Appiah et al., 2008; Kolasa et al., 2016; Koopmanschap et al., 2010; Lasry et al., 2011; Lopert, 2009; Menon et al., 2010; Miot et al., 2012; Mullen, 2004; Tannahill, 2008; Thokala \& Duenas, 2012; Tony et al., 2011; University of York, 2002; Venhorst et al., 2014; Youngkong et al., 2009; Youngkong et al., 2012a. ${ }^{\text {31 }}$ Andreae et al., 2009; Lasry et al., 2011; Lopert, 2009; Youngkong et al., 2009. ${ }^{32}$ Dionne et al., 2009; Golan \& Hansen, 2010; Johnson et al., 2009; Kirby et al., 2008; Mullen, 2004. "33 Marsh et al., 2013; Menon et al., 2010; Venhorst et al., 2014. "34 Baltussen et al., 2006; Browman et al., 2008; Diaby \& Goeree, 2014; Dolan, 2010; Le Gales \& Moatti, 1990; Goetghebeur et al., 2008; Goetghebeur et al. 2010; Goetghebeur et al., 2012; Golan \& Hansen, 2010; Golan \& Hansen, 2012; Gurtner, 2014; Kolasa et al. 2016; Menon et al., 2010; Miot et al., 2012; Noorani et al., 2007; Tony et al., 2011; Tromp \& Baltussen, 2012; Vuorenkoski et al., 2008; Youngkong et al., 2012a. "35 Adunlin et al., 2015; Bowen et al., 2009; Diaby \& Goeree, 2014; Dolan, 
2010; Golan \& Hansen, 2010; Hailey, 2009; Hilgerink et al., 2011; Hummel et al., 2012; Johnson et al., 2009; Martin et al., 2001; Mullen, 2004; Ritrovato et al., 2015; Sloane et al., 2003; University of York, 2002; Venhorst et al., 2014; Vuorenkoski et al., 2008. ${ }^{* 36}$ Johnson et al., 2009; Tromp \& Baltussen, 2012. ${ }^{* 37}$ Dolan, 2010; Kapiriri et al., 2009; Mullen, 2004; Simoens, 2014. ${ }^{* 38}$ Duthie et al., 1999; Johnson et al., 2009. ${ }^{* 39}$ Goetghebeur et al., 2008; Goetghebeur et al., 2010; Goetghebeur et al., 2012; Miot et al., 2012; Tony et al., 2011; Youngkong et al., 2012 a; Youngkong, 2014. ${ }^{* 40}$ Browman et al., 2008. ${ }^{* 41}$ Browman et al., 2008; Golan \& Hansen, 2010; Hofmann, 2005; Johnson et al., 2009; Lehoux \& Williams-Jones, 2007; Martin et al., 2001; Menon et al., 2010; Mullen, 2004; Noorani et al., 2007; Vuorenkoski et al., 2008; Youngkong et al., 2009. ${ }^{* 22}$ Goetghebeur et al., 2008; Goetghebeur et al., 2010; Goetghebeur et al., 2012; Kolasa et al., 2016; Miot et al., 2012; Phillips \& Costa, 2007: Tony et al., 2011. *43 Golan \& Hansen, 2010; Johnson et al., 2009: Kapiriri et al., 2009; Lasry et al., 2011; Martin et al., 2001; Meagher, 2010; Mullen, 2004; Wilson et al., 2006: Wirtz et al., 2005. ${ }^{* 44}$ Gibson et al., 2005; Irving et al., 2010; Lopert, 2009. "45 Duthie et al., 1999; Irving et al., 2010; Youngkong et al., 2009. .46 Goetghebeur et al., 2008; Goetghebeur et al., 2010; Goetghebeur et al., 2012; Kirby et al., 2008; Miot et al., 2012; Tony et al., 2011. "47 Diaby \& Goeree, 2014; Vuorenkoski et al., 2008. ${ }^{* 48}$ Johnson et al., 2009; Kolasa et al., 2016; Martin et al., 2001; Noorani et al., 2007; Vuorenkoski et al., 2008. "49 Hofmann, 2005; Noorani et al., 2007. "50 Goetghebeur et al., 2008; Goetghebeur et al., 2010; Goetghebeur et al., 2012; Hofmann, 2005; Lehoux \&Williams-Jones, 2007; Miot et al., 2012; Tony et al., 2011. "51 Golan \& Hansen, 2010; Johnson et al., 2009; Kapiriri et al., 2009; Mullen, 2004. ${ }^{* 52}$ Browman et al., 2008; Dionne et al., 2015; Mullen, 2004. ${ }^{* 33}$ Kirby et al., 2008; Marsh et al., 2013; Wilson et al., 2007. ${ }^{* 54}$ Diaby \& Goeree, 2014; Golan \& Hansen, 2012; Hofmann, 2005; Johnson et al., 2009; Lehoux \&Williams-Jones, 2007; Tannahill, 2008; Thokala \& Duenas, 2012; Venhorst et al., 2014; Vuorenkoski et al., 2008;Youngkong et al., 2012b. *55 Mullen, 2004; Tannahill, 2008. ${ }^{* 56}$ Diaby \& Goeree, 2014; Hofmann, 2005; Lehoux \& Williams-Jones, 2007 *57 Goetghebeur et al, 2008; Goetghebeur et al. 2010; Goetghebeur et al, 2012; Golan \& Hansen, 2010; Miot et al. 2012; Tony et al., 2011. *58 Hofmann, 2005; Lehoux \& Williams-Jones, 2007. ${ }^{* 59}$ Diaby \& Goeree, 2014; Hofmann, 2005; Lehoux \& Williams-Jones, 2007. " ${ }^{60}$ Johnson et al., 2009; Lehoux \& Williams-Jones, 2007; Noorani et al., 2007; Ritrovato et al., 2015. "61 Bowen et al., 2009; Tromp \& Baltussen, 2012; Venhorst et al., 2014. "62 Nobre et al., 1999; Vuorenkoski et al., 2008. ${ }^{* 63}$ Ghandour et al., 2015; Johnson et al., 2009; Mullen, 2004; Venhorst et al., 2014; Wilson et al.,2007. ${ }^{* 64}$ Tannahill, 2008; Wilson et al., 2007."65 Ghandour et al., 2015; Johnson et al., 2009: Kapiriri et al., 2009.*66 Johnson et al., 2009; Tannahill, 2008. *67 Shin et al., 2009; Wilson et al., 2006. ${ }^{* 68}$ Goetghebeur et al., 2008; Goetghebeur et al., 2010; Goetghebeur et al., 2012; Miot et al., 2012; Tony et al., 2011; Youngkong et al., 2012b. ${ }^{* 69}$ Airoldi et al., 2014; Bowen et al., 2009; Diaby \& Goeree, 2014; Ghandour et al., 2015; Gurtner, 2014; Johnson et al., 2009; Kapiriri et al., 2009; Menon et al., 2010; Ritrovato et al., 2015; Wirtz et al., 2005. ${ }^{~ 70}$ Dionne et al., 2015; Le Gales \& Moatti, 1990. ${ }^{* 71}$ Lehoux \& Williams-Jones, 2007; Simoens, 2014. "72 Duthie et al., 1999; Ghandour et al., 2015; Gibson et al., 2006. "73 Kapiriri et al., 2009; Saarni et al., 2008; Tannahill, 2008; Tromp \& Baltussen, 2012; Vuorenkoski et al. 2008. "74 Johnson et al., 2009; Ritrovato et al. 2015 *75 Kirby et al., 2008; Meagher, 2010; Tannahill, 2008. "76 Lasry et al., 2011; Mullen, 2004; Wilson et al., 2006; Youngkong et al., 2009. ${ }^{* 77}$ Asante \& Zwi, 2009; Noorani et al., 2007. ${ }^{* 78}$ Dionne et al., 2009; Duthie et al., 1999; Ghandour et al., 2015; Gibson et al., 2006; Golan \& Hansen, 2010; Hofmann, 2005; Johnson et al., 2009; Lehoux \& Williams-Jones, 2007; Mullen, 2004; University of York, 2002; Venhorst et al., 2014; Vuorenkoski et al., 2008; Wilson et al., 2006; Wirtz et al., 2005. ${ }^{* 79}$ Mullen, 2004; Youngkong et al., 2009. ${ }^{* 80}$ Dionne et al., 2015; Duthie et al., 1999; Johnson et al., 2009; Youngkong et al., 2009. ${ }^{* 81}$ Cho \& Kim, 2003; Nobre et al., 1999. ${ }^{* 82}$ Airoldi et al., 2014; Mullen, 2004. ${ }^{* 83}$ Asante \& Zwi, 2009; Dionne et al., 2015; Golan \& Hansen, 2010; Golan \& Hansen, 2012; Kirby et al., 2008; Lasry et al., 2011; Lehoux \& Williams-Jones, 2007; Menon et al., 2010; Mitton et al., 2006; Mullen, 2004; Saarni et al., 2008; Tannahill, 2008; Thokala \& Duenas, 2012; University of York, 2002; Wilson et al., 2007; Youngkong et al., 2009. .84 Baltussen et al., 2006; Baltussen et al., 2007; Defechereux et al., 2012; Diaby \& Lachaine, 2011; Youngkong et al., 2009. ${ }^{* 85}$ Kapiriri et al., 2009; Golan \& Hansen, 2010; Tannahill, 2008. ${ }^{* 86}$ Lasry et al., 2011; Lehoux \& Williams-Jones, 2007; Mullen, 2004; Vuorenkoski et al., 2008. " ${ }^{87}$ Duthie et al., 1999; Johnson et al., 2009; Nobre et al., 1999; Youngkong et al., 2009. *88 Youngkong et al., 2012a; Youngkong. 2014. ${ }^{* 9}$ Hofmann, 2005; Lehoux \& Williams-Jones, 2007. ${ }^{* 00}$ Goetghebeur et al., 2008; Goetghebeur et al., 2010; Goetghebeur et al., $2012 ;$ Miot et al., 2012; Tony et al., 2011. ${ }^{* 11}$ Goetghebeur et al., 2010; Saarni et al., 2008; Tannahill, 2008. "92 Kirby et al., 2008; Menon et al., 2010. "93 Baeten et al., 2010; Baltussen et al., 2006; Baltussen et al., 2007; Jehu-Appiah et al., 2008; Youngkong et al., 2009. "94 Diaby \& Goeree, 2014; Jehu-Appiah et al., 2008; Kapiriri et al., 2009; Mullen, 2004. *95 Lehoux \& Williams-Jones, 2007; Tannahill, 2008 . 96 Golan \& Hansen, 2010; Menon et al., 2010; Tannahill, 2008. ${ }^{* 97}$ Ghaffar, 2009; Tannahill, 2008; Walton et al., 2007. ${ }^{* 98}$ Hofmann, 2005; Lehoux \& Williams-Jones, 2007. ${ }^{* 99}$ Diaby \& Goeree, 2014; Johnson et al., 2009. ${ }^{* 100}$ Hofmann, 2005; Lehoux \& Williams-Jones, 2007. ${ }^{101}$ Le Gales \& Moatti, 1990; Hofmann, 2005; Johnson et al., 2009; Lasry et al., 2011; Lehoux \& Williams-Jones, $2007 ;$ Noorani et al., 2007; Tannahill, 2008. ${ }^{*} 102$ Bowen et al., 2009; Johnson et al., 2009. ${ }^{* 103}$ Andreae et al., 2009; Kirby et al., 2008. ${ }^{* 104}$ Ghaffar, 2009; Wirtz et al., 2005. ${ }^{* 105}$ Ghaffar, 2009; Walton et al., 2007. ${ }^{*} 106$ Honoré et al., 2010; Walton

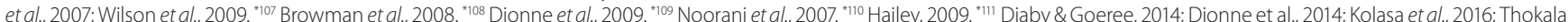
\& Duenas, 2012. ${ }^{* 12}$ Andreae et al., 2009; Kirby et al., 2008; Mitton et al., 2006. ${ }^{* 113}$ Andreae et al., 2009; Browman et al., $2008 ;$ Mitton et al., $2006 .{ }^{* 114}$ Walton et al., $2007 .{ }^{* 115}$ Walton et al., 2007. ${ }^{* 116}$ Le Gales \& Moatti, 1990. *117 Walton et al., 2007.*118 Browman et al., 2008; Noorani et al., 2007. *119 Diaby \& Goeree, 2014; Dolan, 2010; Gibson et al., 2006. *120 Diaby \& Goeree, 2014; Goetghebeur et al., 2010; Wilson et al., 2009. "121 Goetghebeur et al., 2010; Wilson et al., 2009. "122 Golan \& Hansen, 2010; Noorani et al., 2007; Youngkong et al., 2009. "123 Browman et al., 2008; Wilson et al., 2009. *124 Golan \& Hansen, 2010; Vuorenkoski et al., 2008.

(4 critérios); qualidade e incerteza da evidência (4 critérios); implementação da intervenção (5 critérios); benefícios para a sociedade (5 critérios); benefícios para o sistema de saúde público (5 critérios); benefícios para a indústria (1 critério); e outros (3 critérios).

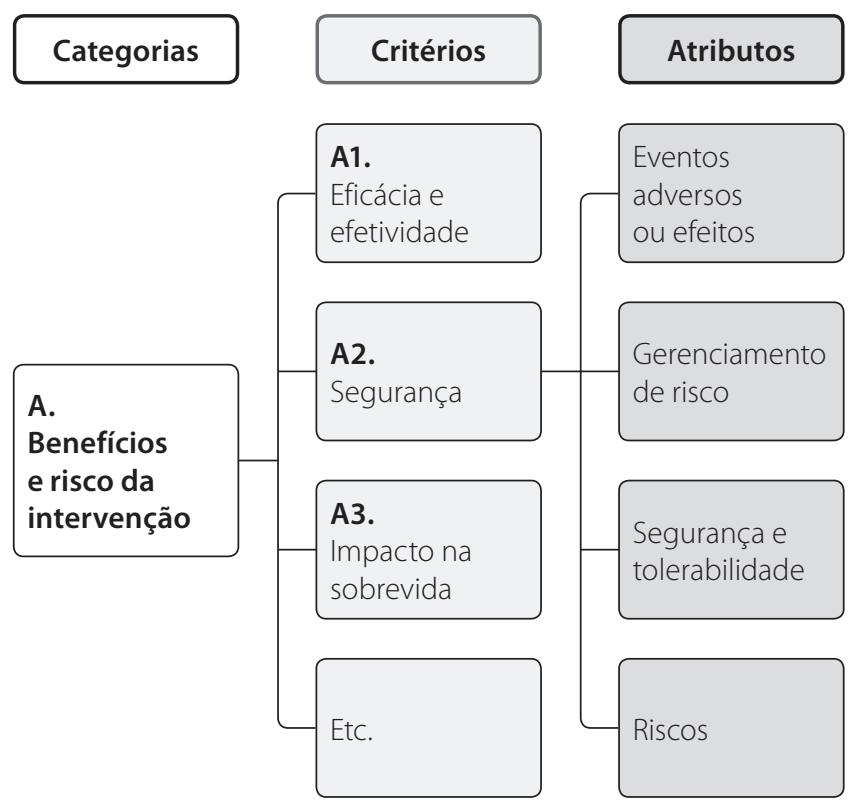

Figura 2. Categorização dos termos extraídos da literatura.
Alguns critérios e atributos encontrados eram muito específicos de determinadas tecnologias e foram excluídos da síntese. Por exemplo, os atributos "mortalidade materna" e "qualidade dos serviços de cuidado materno" eram específicos para programa de segurança materna (Youngkong et al., 2009).

Determinados critérios apresentaram grande variedade de atributos (forma de mensuração do critério), como: requisitos organizacionais e implementação (26 atributos); impacto social e vulnerabilidade (25 atributos); eficácia e efetividade (22 atributos); desfechos centrados no paciente (14 atributos); aspectos morais e éticos (12 atributos); interesses e pressões dos interessados (stakeholders) (12 atributos) e força da evidência (10 atributos).

Alguns atributos foram citados em muitos estudos, como: custo-efetividade (28 referências); segurança e tolerabilidade (20 referências); impacto orçamentário para o sistema/plano de saúde (19 referências); gravidade da doença (18 referências); equidade (17 referências); efetividade (16 referências); custos (16 referências); acesso (14 referências); eficácia/efetividade (12 referências); e alternativas de tratamento disponíveis (11 referências).

A Tabela 1 apresenta as categorias e os principais critérios e atributos identificados nesta revisão. A Tabela 2 apresenta a lista de estudos incluídos na revisão rápida.

\section{Discussão}

O MCDA nas decisões de incorporação de tecnologias em saúde tem o potencial de tornar a decisão mais transparente, 
Tabela 2. Lista de estudos incluídos na revisão rápida

\begin{tabular}{|c|c|c|c|}
\hline Primeiro autor, ano & Título & $\begin{array}{l}\text { Desenho do } \\
\text { estudo }\end{array}$ & Origem da busca \\
\hline Adunlin, 2015 & Multicriteria decision analysis in oncology & Estudo de caso & Busca PubMed \\
\hline Baeten, 2010 & $\begin{array}{l}\text { Incorporating equity-efficiency interactions in cost-effectiveness } \\
\text { analysis-three approaches applied to breast cancer control }\end{array}$ & Estudo de caso & Busca PubMed \\
\hline Baltussen, 2006 & Towards a multi-criteria approach for priority setting: an application to Ghana & Estudo de caso & Literatura cinzenta \\
\hline Baltussen, 2007 & $\begin{array}{l}\text { Priority setting using multiple criteria: should a lung } \\
\text { health programme be implemented in Nepal? }\end{array}$ & Estudo de caso & Busca PubMed \\
\hline Broekhuizen, 2015 & $\begin{array}{l}\text { Estimating the value of medical treatments to patients } \\
\text { using probabilistic multi criteria decision analysis }\end{array}$ & Estudo de caso & Busca PubMed \\
\hline Defechereux, 2012 & Health care priority setting in Norway a multicriteria decision analysis & Estudo de caso & Busca PubMed \\
\hline Diaby, 2014 & $\begin{array}{l}\text { Multi-criteria decision analysis for health technology assessment } \\
\text { in Canada: insights from an expert panel discussion }\end{array}$ & Estudo de caso & Busca PubMed \\
\hline Dionne, 2015 & $\begin{array}{l}\text { Developing a multi-criteria approach for drug reimbursement } \\
\text { decision making: an initial step forward }\end{array}$ & Estudo de caso & Busca PubMed \\
\hline Ghandour, 2015 & $\begin{array}{l}\text { Priority setting for the prevention and control of cardiovascular diseases: } \\
\text { multi-criteria decision analysis in four eastern Mediterranean countries }\end{array}$ & Estudo de caso & Busca PubMed \\
\hline Goetghebeur, 2008 & $\begin{array}{l}\text { Evidence and Value: Impact on DEcisionMaking - the } \\
\text { EVIDEM framework and potential applications }\end{array}$ & $\begin{array}{l}\text { Revisão narrativa } \\
\text { e Estudo de caso }\end{array}$ & Busca PubMed \\
\hline Goetghebeur, 2012 & $\begin{array}{l}\text { Bridging health technology assessment (HTA) and efficient health } \\
\text { care decision making with multicriteria decision analysis (MCDA): } \\
\text { applying the EVIDEM framework to medicines appraisal }\end{array}$ & Estudo de caso & Busca PubMed \\
\hline $\begin{array}{l}\text { Greef-van der } \\
\text { Sandt, } 2015\end{array}$ & $\begin{array}{l}\text { A quantitative benefit-risk assessment approach to improve decision making } \\
\text { in drug development: application of a multicriteria decision analysis model } \\
\text { in the development of combination therapy for overactive bladder }\end{array}$ & Estudo de caso & Busca PubMed \\
\hline
\end{tabular}

\begin{tabular}{|c|c|c|c|}
\hline Guindo, 2012 & $\begin{array}{l}\text { From efficacy to equity: literature review of decision criteria for } \\
\text { resource allocation and healthcare decision making }\end{array}$ & Revisão narrativa & Literatura cinzenta \\
\hline Gurtner, 2014 & $\begin{array}{l}\text { Making the right decisions about new technologies: a } \\
\text { perspective on criteria and preferences in hospitals }\end{array}$ & Estudo de caso & Literatura cinzenta \\
\hline Jehu-Appiah, 2008 & $\begin{array}{l}\text { Balancing equity and efficiency in health priorities in } \\
\text { Ghana: the use of Multicriteria Decision Analysis. }\end{array}$ & Estudo de caso & Literatura cinzenta \\
\hline Kolasa, 2016 & $\begin{array}{l}\text { Potential impact of the implementation of multiple-criteria decision analysis } \\
\text { (MCDA) on the Polish pricing and reimbursement process of orphan drugs }\end{array}$ & $\begin{array}{l}\text { Revisão } \\
\text { sistemática e } \\
\text { estudo de caso }\end{array}$ & Busca PubMed \\
\hline Mühlbacher, 2015 & $\begin{array}{l}\text { Making Good Decisions in Healthcare with Multi-Criteria Decision Analysis: } \\
\text { The Use, Current Research and Future Development of MCDA }\end{array}$ & Revisão narrativa & Busca PubMed \\
\hline Nobre, 1999 & Multi-criteria decision making - an approach to setting priorities in health care & Estudo de caso & Busca PubMed \\
\hline Ritrovato, 2015 & $\begin{array}{l}\text { Decision-oriented health technology assessment: one step forward } \\
\text { in supporting the decision-making process in hospitals }\end{array}$ & Estudo de caso & Busca PubMed \\
\hline Simoens, 2014 & Health technologies for rare diseases: does conventional HTA still apply? & Revisão narrativa & Busca PubMed \\
\hline Thokala, 2012 & Multiple criteria decision analysis for health technology assessment & Estudo de caso & Busca PubMed \\
\hline Thokala, 2016 & $\begin{array}{l}\text { Multiple Criteria Decision Analysis for Health Care Decision Making - An } \\
\text { Introduction: Report } 1 \text { of the ISPOR MCDA Emerging Good Practices Task Force }\end{array}$ & Revisão narrativa & Busca PubMed \\
\hline Tony, 2011 & $\begin{array}{l}\text { Bridging health technology assessment (HTA) with multicriteria } \\
\text { decision analyses (MCDA): field testing of the EVIDEM framework } \\
\text { for coverage decisions by a public payer in Canada }\end{array}$ & Estudo de caso & Busca PubMed \\
\hline Tromp, 2012 & $\begin{array}{l}\text { Mapping of multiple criteria for priority setting of health } \\
\text { interventions: an aid for decision makers }\end{array}$ & Revisão narrativa & Busca PubMed \\
\hline Wahlster, 2015 & $\begin{array}{l}\text { Balancing costs and benefits at different stages of medical innovation: } \\
\text { a systematic review of Multi-Criteria Decision Analysis (MCDA) }\end{array}$ & $\begin{array}{l}\text { Revisão } \\
\text { sistemática }\end{array}$ & Busca PubMed \\
\hline Youngkong, 2012 & $\begin{array}{l}\text { Multicriteria decision analysis for including health interventions in } \\
\text { the universal health coverage benefit package in Thailand }\end{array}$ & Estudo de caso & Busca PubMed \\
\hline Youngkong, 2014 & Application of HTA research on policy decision-making & Estudo de caso & Busca PubMed \\
\hline
\end{tabular}


coerente, auditável e defensável (Youngkong, 2014; Thokala \& Duenas, 2012). Permite que os tomadores de decisão tenham acesso a uma lista de critérios predefinidos, criando uma estrutura reprodutível nos diversos processos. Apresentar os critérios de forma clara e objetiva possibilita ao decisor valorar a importância de cada um e deliberar de modo mais realista e assertivo.

Existe dúvida entre a realização do MCDA com número restrito de critérios ou a utilização de uma gama mais ampla na avaliação. Ao utilizar um número restrito de critérios, a matriz torna-se mais simples e objetiva, porém critérios relevantes como equidade e aspectos éticos podem ser desconsiderados numa tomada de decisão. Já uma matriz mais ampla e com variedade de critérios tende a ser mais complexa e de difícil entendimento. Logo, seu uso pode se tornar restrito, uma vez que o tempo gasto para sua compreensão e julgamento pode inviabilizar uma decisão ágil.

O presente estudo elencou uma lista abrangente de critérios, e diversos deles apresentavam menor peso numa tomada de decisão. Nesse caso, a importância de incluí-los numa deliberação, em teoria, deve-se ao fato de que, ao se somarem vários critérios menos relevantes, eles poderiam ter peso suficiente para alterar os resultados de uma avaliação. Dessa forma, não apenas os critérios mais científicos ou quantitativos seriam considerados, como eficácia, segurança e custo-efetividade, uma vez que eles são fundamentais numa tomada de decisão e são os primeiros a serem incluídos. No estudo realizado por Goetghebeur et al. (2012), os participantes afirmaram que a integração de vários critérios de ATS na matriz de MCDA permitiu melhor organização e síntese dos dados, facilitando a transferência de conhecimento (Goetghebeur et al., 2012).

Outra discussão pertinente é entre utilizar um conjunto de critérios genéricos e fixos que se apliquem a quaisquer decisões de incorporação ou criar critérios mais específicos como os para doenças raras ou para equipamentos médicos. Alguns critérios considerados operacionais apenas em um contexto muito restrito não foram incluídos na seleção realizada, no entanto eles poderiam ser utilizados em modelos de MCDA específicos para determinadas atuações ou áreas. Tervonen et al. (2015) (Tervonen et al., 2015), por exemplo, aplicaram o MCDA na seleção das estatinas na prevenção primária da doença cardiovascular e definiram seis critérios a partir dos desfechos da doença (todas as causas de mortalidade, derrame não fatal, infarto do miocárdio não fatal, mialgia, elevação de transaminases e elevação da CK).

Duas áreas em que a utilização de MCDA tem se estruturado são a oncologia e nas doenças raras. O uso do MCDA na oncologia é uma decisão complexa, pois exige tomadas de decisões completamente diferentes de acordo com as características específicas de cada tumor, diagnóstico e tratamento da doença. A oncologia é um desafio para a saúde, pois, à medida que a compreensão da complexidade do câncer aumenta, o desenvolvimento de novas tecnologias e os custos também são crescentes (Adunlin et al., 2015). Nesse caso, a elaboração de uma ferramenta ampla de auxílio à tomada de decisão com o uso do MCDA pode ser insuficiente para atender aos anseios dessa área de saúde, com características tão específicas.

O caso das doenças raras deve ser considerado de forma diferenciada das doenças mais prevalentes. As doenças raras possuem características únicas, como: preços elevados de medicamentos, monopólio, inexistência de outros tratamentos, gravidade elevada, baixa qualidade dos estudos e prevalência baixa da doença. Nesse caso, Hughes-Wilson et al. (2012) sugerem a elaboração de um sistema de avaliação de tecnologias para doenças raras com múltiplos critérios de avaliação específicos nesse contexto e com uma forma de alocação de pesos específica. Assim, essa ferramenta poderia auxiliar gestores governamentais na tomada de decisão acerca do tratamento das doenças raras, uma vez que essas tecnologias não seriam custo-efetivas quando avaliadas pelos mesmos critérios utilizados pelas outras tecnologias mais usuais (Hughes-Wilson et al., 2012).

As doenças raras geram alta comoção da sociedade. Em alguns estudos, a população afirmou estar disposta a pagar mais por uma doença que é rara ou muito grave (Hughes-Wilson et al., 2012). Por outro lado, existe um paradoxo relacionado às doenças raras, uma vez que essas doenças acometem pequena quantidade de pessoas, porém existem em grande número (Melnikova, 2012). Dessa forma, é preciso avaliar quanto a sociedade está disposta a pagar pelo tratamento dessas doenças e o custo de oportunidade, pois esses recursos vão representar a perda em algum outro setor, expressando os trade-offs enfrentados pelo gestor. Ou seja, os recursos empregados na incorporação de tecnologias para o tratamento de alguma doença rara, para uma pequena quantidade de pessoas, deixarão de ser empregados para outros fins em grande número de indivíduos na saúde pública (Prado, 2015).

Uma dificuldade encontrada na seleção dos critérios e na identificação dos atributos foi a redundância de alguns termos. Nesse caso, a origem e o significado do termo foi revisto e reanalisado a fim de esclarecer potenciais divergências. Alguns termos foram agrupados, pois eram muito semelhantes e apresentavam um mesmo sentido. A forma ou o estilo como as palavras são utilizadas determinam seu significado (wording). Guindo et al. (2012) identificaram uma variedade de terminologias em sua revisão da literatura e afirmaram que a classificação dos critérios foi limitada pela interpretação subjetiva dos autores dos estudos e pela dificuldade em harmonizar os diferentes tipos de processos decisórios (Guindo et al., 2012). Observa-se grande variedade de critérios selecionados na revisão. Assim, é preciso analisar se essa quantidade é 
adequada ou extrapola o necessário para o tipo de decisão. Recomenda-se analisar essa lista de critérios com um grupo de especialistas e realizar uma proposta com os termos mais importantes para a instituição. Outra questão importante seria averiguar se diferentes tecnologias como medicamentos, procedimentos e dispositivos médicos poderiam gerar listas específicas de critérios ou se cenários diferentes como oncologia e doenças raras devem possuir listas diferentes (Ferreli et al., 2014).

\section{Conclusão}

Este estudo apresenta a análise de decisão multicritérios como uma proposta a ser utilizada para compor uma importante ferramenta no auxílio à tomada de decisões, no sentido de sinalizar aos decisores de instâncias como a Conitec os diversos critérios que podem ser considerados numa recomendação de incorporação ou exclusão de tecnologias no SUS. Sugere-se que a lista com os critérios não deva ser fixa, mas livre para que sejam ou não considerados, conforme o tipo de demanda analisada. O objetivo principal de se utilizar a MCDA nesse processo é de deixar a decisão mais transparente, compensatória e eficiente.

\section{Referências bibliográficas}

Adunlin G, Diaby V, Montero AJ, Xiao H. Multicriteria decision analysis in oncology. Health Expect. 2015;18(6):1812-26.

Airoldi M, Morton A, Smith JA, Bevan G. STAR - people-powered prioritization: a 21st-century solution to allocation headaches. Med Decis Making. 2014;34(8):965-75.

Andreae MC, Lamarand KE, Abraham L, Freed GL. Basis for immunization recommendations among countries of the World Health Organization European region. Hum Vaccin. 2009;5(11):754-60.

Asante AD, Zwi AB. Factors influencing resource allocation decisions and equity in the health system of Ghana. Public Health. 2009;123(5):371-7.

Baeten SA, Baltussen RM, Uyl-de Groot CA, Bridges J, Niessen LW. Incorporating equity-efficiency interactions in cost-effectiveness analysis-three approaches applied to breast cancer control. Value Health. 2010;13(5):573-9.

Baltussen R, Niessen L. Priority setting of health interventions: the need for multi-criteria decision analysis. Cost Eff Resour Alloc. 2006;4:14.

Baltussen R, Stolk E, Chisholm D, Aikins M. Towards a multi-criteria approach for priority setting: an application to Ghana. Health Econ. 2006;15(7):689-96.

Baltussen R, ten Asbroek AH, Koolman X, Shrestha N, Bhattarai P, Niessen LW. Priority setting using multiple criteria: should a lung health programme be implemented in Nepal? Health Policy Plan. 2007;22(3):178-85.

Belton V, Stewart T. Multiple criteria decision analysis: an integrated approach. New York: Springer Science \& Business Media; 2002.

Bots PW, Hulshof JA. Designing multi-criteria decision analysis processes for priority setting in health policy. J Multi-Crit Decis Anal. 2000;9(1-3):56-75.

Bowen S, Erickson T, Martens PJ, Crockett S. More than "using research": the real challenges in promoting evidence-informed decision-making. Healthc Policy. 2009;4(3):87-102.
Brasil. Casa Civil. Lei no 12.401, de 28 de abril de 2011. Altera a Lei no 8.080, de 19 de setembro de 1990, para dispor sobre a assistência terapêutica e incorporação de tecnologia em saúde no âmbito do Sistema Único de Saúde - SUS. Diário Oficial da República Federativa do Brasil, Brasília (DF), 2011a; Seção 1.

Brasil. Casa Civil. Decreto no 7.646, de 21 de dezembro de 2011. Dispõe sobre a Comissão Nacional de Incorporação de Tecnologias no Sistema Único de Saúde e sobre o processo administrativo para incorporação, exclusão e alteração de tecnologias em saúde pelo Sistema Único de Saúde SUS, e dá outras providências. Diário Oficial da República Federativa do Brasil, Brasília (DF), 2011b.

Broekhuizen H, Groothuis-Oudshoorn CG, Hauber AB, Jansen JP, IJzerman MJ. Estimating the value of medical treatments to patients using probabilistic multi criteria decision analysis. BMC Med Inform Decis Mak. 2015;15:102.

Browman GP, Manns B, Hagen N, Chambers CR, Simon A, Sinclair S. 6-STEPPPS: A Modular Tool to Facilitate Clinician Participation in Fair Decisions for Funding New Cancer Drugs. J Oncol Pract. 2008;4(1):2-7.

Cho KT, Kim SM. Selecting medical devices and materials for development in Korea: the analytic hierarchy process approach. Int J Health Plann Manage. 2003;18(2):161-74.

Defechereux T, Paolucci F, Mirelman A, Youngkong S, Botten G, Hagen TP, et al. Health care priority setting in Norway a multicriteria decision analysis. BMC Health Serv Res. 2012;12(1):39.

de Greef-van der Sandt I, Newgreen D, Schaddelee M, Dorrepaal C, Martina $\mathrm{R}$, Ridder A, et al. A quantitative benefit-risk assessment approach to improve decision making in drug development: Application of a multicriteria decision analysis model in the development of combination therapy for overactive bladder. Clin Pharmacol Ther. 2016:99(4):442-51.

Devlin N, Sussex J. Incorporating multiple criteria in HTA: methods and processes. London: Office of Health Economics; 2011.

Dhalla I, Laupacis A. Moving from opacity to transparency in pharmaceutical policy. Can Med Assoc J. 2008;178:428-31.

Diaby V, Goeree R. How to use multi-criteria decision analysis methods for reimbursement decision-making in healthcare: a step-by-step guide. Expert Rev Pharmacoecon Outcomes Res. 2014;14(1):81-99.

Diaby V, Goeree R, Hoch J, Siebert U. Multi-criteria decision analysis for health technology assessment in Canada: insights from an expert panel discussion. Expert Rev Pharmacoecon Outcomes Res. 2015;15(1):13-9.

Diaby V, Lachaine J. An application of a proposed framework for formulary listing in low-income countries: the case of Côte d'Ivoire. Appl Health Econ Health Policy. 2011;9(6):389-402.

Dionne F, Mitton C, Dempster B, Lynd LD. Developing a multi-criteria approach for drug reimbursement decision making: an initial step forward. J Popul Ther Clin Pharmacol. 2015;22(1):e68-77.

Dionne F, Mitton C, Smith N, Donaldson C. Evaluation of the impact of program budgeting and marginal analysis in Vancouver Island Health Authority. J Health Serv Res Policy. 2009;14(4):234-42.

Dodgson JS, Spackman M, Pearman A, Phillips LD. Multi-criteria analysis: a manual. Department for Communities and Local Government. London; 2009.

Dolan JG. Multi-criteria clinical decision support: A primer on the use of multiple criteria decision making methods to promote evidence-based, patient-centered healthcare. Patient. 2010;3(4):229-248.

Duthie T, Trueman P, Chancellor J, Diez L. Research into the use of health economics in decision making in the United Kingdom - Phase II. Is health economics 'for good or evil'? Health Policy. 1999;46(2):143-57.

Ferreli C, Atzori L, Manunza F, Pau M, Caddori A. Thalidomide-induced granuloma annulare. G Ital Dermatol Venereol. 2014;149(3):329-33. 
Ghaffar A. Setting research priorities by applying the combined approach matrix. Indian J Med Res. 2009;129(4):368-75.

Ghandour R, Shoaibi A, Khatib R, Abu Rmeileh N, Unal B, Sözmen K, et al. Priority setting for the prevention and control of cardiovascular diseases: multi-criteria decision analysis in four eastern Mediterranean countries. Int J Public Health. 2015;60 Suppl 1:S73-81.

Gibson JL, Martin DK, Singer PA. Evidence, economics and ethics: resource allocation in health services organizations. Healthc Q. 2005;8(2):50-9.

Gibson J, Mitton C, Martin D, Donaldson C, Singer P. Ethics and economics: does programme budgeting and marginal analysis contribute to fair priority setting? J Health Serv Res Policy. 2006;11(1):32-7.

Goetghebeur MM, Wagner M, Khoury H, Levitt RJ, Erickson LJ, Rindress D. Evidence and value: impact on DEcisionMaking - the EVIDEM framework and potential applications. BMC Health Serv Res. 2008;8:270.

Goetghebeur MM, Wagner M, Khoury H, Rindress D, Grégoire JP, Deal C. Combining multicriteria decision analysis, ethics and health technology assessment: applying the EVIDEM decision-making framework to growth hormone for Turner syndrome patients. Cost Eff Resour Alloc. 2010;8:4.

Goetghebeur MM, Wagner M, Khoury H, Levitt RJ, Erickson LJ, Rindress D. Bridging health technology assessment (HTA) and efficient health care decision making with multicriteria decision analysis (MCDA): applying the EVIDEM framework to medicines appraisal. Med Decis Making. 2012;32(2):376-88.

Golan O, Hansen P. A new decision-support framework for prioritization of new health technologies. University of Otago: The 'value for money' chart; 2010.

Golan O, Hansen P. Which health technologies should be funded? A prioritization framework based explicitly on value for money. Isr J Health Policy Res. 2012;1(1):44.

Guindo LA, Wagner M, Baltussen R, Rindress D, van Til J, Kind P, et al. From efficacy to equity: literature review of decision criteria for resource allocation and healthcare decisionmaking. Cost Eff Resour Alloc. 2012;10(9).

Gurtner S. Making the right decisions about new technologies: a perspective on criteria and preferences in hospitals. Health Care Manage Rev. 2014;39(3):245-54.

Hailey D. A preliminary survey on the influence of rapid health technology assessments. Int J Technol Assess Health care. 2009;25(3):415-8.

Hilgerink MP, Hummel MJ, Manohar S, Vaartjes SR, Ijzerman MJ. Assessment of the added value of the Twente Photoacoustic Mammoscope in breast cancer diagnosis. Med Devices (Auckl). 2011;4:107-15.

Hofmann B. Toward a procedure for integrating moral issues in health technology assessment. Int J Technol Assess Health Care. 2005;21(3):312-8.

Honoré PA, Fos PJ, Smith T, Riley M, Kramarz K. Decision science: a scientific approach to enhance public health budgeting. J Public Health Manag Pract. 2010;16(2):98-103.

Hughes-Wilson W, Palma A, Schuurman A, Simoens S. Paying for the Orphan Drug System: break or bend? Is it time for a new evaluation system for payers in Europe to take account of new rare disease treatments? Orphanet J Rare Dis. 2012;7:74.

Hummel JM, Boomkamp IS, Steuten LM, Verkerke BG, ljzerman MJ. Predicting the health economic performance of new non-fusion surgery in adolescent idiopathic scoliosis. J Orthop Res. 2012;30(9):1453-8.

Irving MJ, Tong A, Rychetnik L, Walker RG, Frommer MS, Craig JC. Nephrologists' perspectives on the effect of guidelines on clinical practice: a semistructured interview study. Am J Kidney Dis. 2010;55(2):241-9.
Jehu-Appiah C, Baltussen R, Acquah C, Aikins M, d'Almeida SA, Bosu WK, et al. Balancing equity and efficiency in health priorities in Ghana: the use of multicriteria decision analysis. Value Health. 2008;11(7):1081-7.

Johnson AP, Sikich NJ, Evans G, Evans W, Giacomini M, Glendining M, et al. Health technology assessment: a comprehensive framework for evidence-based recommendations in Ontario. Int J Technol Assess Health Care. 2009;25(2):141-50.

Kapiriri L, Norheim OF, Martin DK. Fairness and accountability for reasonableness. Do the views of priority setting decision makers differ across health systems and levels of decision making? Soc Sci Med. 2009;68(4):766-73.

Kirby J, Somers E, Simpson C, McPhee J. The public funding of expensive cancer therapies: synthesizing the "3Es" - evidence, economics, and ethics. Organ Ethic. 2008;4(2):97-108.

Kolasa K, Zwolinski KM, Kalo Z, Hermanowski T. Potential impact of the implementation of multiple-criteria decision analysis (MCDA) on the Polish pricing and reimbursement process of orphan drugs. Orphanet J Rare Dis. 2016;11(1):23.

Koopmanschap MA, Stolk EA, Koolman X. Dear policy maker: have you made up your mind? A discrete choice experiment among policy makers and other health professionals. Int J Technol Assess Health Care. 2010;26(2):198-204.

Lasry A, Carter MW, Zaric GS. Allocating funds for HIV/AIDS: a descriptive study of KwaDukuza, South Africa. Health Policy Plan. 2011;26(1):33-42.

Le Gales C, Moatti JP. Searching for consensus through multi-criteria decision analysis. Assessment of screening strategies for hemoglobinopathies in southeastern France. Int J Technol Assess Health Care. 1990;6(3):430-49.

Lehoux P, Williams-Jones B. Mapping the integration of social and ethical issues in health technology assessment. Int J Technol Assess Health Care. 2007;23(1):9-16.

Lopert R. Evidence-based decision-making within Australia's pharmaceutical benefits scheme. Issue Brief (Common Fund). 2009;60:1-13.

Marsh K, Dolan P, Kempster J, Lugon M. Prioritizing investments in public health: a multi-criteria decision analysis. J Public Health (Oxf). 2013;35(3):460-6.

Martin DK, Pater JL, Singer PA. Priority-setting decisions for new cancer drugs: a qualitative case study. Lancet. 2001;358(9294):1676-81.

Meagher T. MUHC clinical activity priority setting A4R and beyond. Presented at the 8th Biennial Conference of the International Society on Priorities in Health Care. Boston; 2010.

Melnikova I. Rare diseases and orphan drugs. Nat Rev Drug Discov. 2012;11(4):267-8.

Menon D, Stafinski T, McCabe C. To fund or not to fund: a generalized decision-making model for health care resource allocation. Presented at the 8th Biennial Conference of the International Society on Priorities in Health Care. Boston; 2010.

Miot J, Wagner M, Khoury H, Rindress D, Goetghebeur MM. Field testing of a multicriteria decision analysis (MCDA) framework for coverage of a screening test for cervical cancer in South Africa. Cost Eff Resour Alloc. 2012;10(1):2.

Mitton C, Mackenzie J, Cranston L, Teng F. Priority setting in the Provincial Health Services Authority: case study for the 2005/06 planning cycle. Healthc Policy. 2006;2(1):91-106.

Mühlbacher AC, Kaczynski A. Making good decisions in healthcare with multi-criteria decision analysis: the use, current research and future development of MCDA. Appl Health Econ Health Policy. 2015:1-12.

Mullen PM. Quantifying priorities in healthcare: transparency or illusion? Health Serv Manage Res. 2004;17(1):47-58. 
Nobre FF, Trotta LT, Gomes LF. Multi-criteria decision making - an approach to setting priorities in health care. Stat Med. 1999;18(23):3345-54.

Noorani HZ, Husereau DR, Boudreau R, Skidmore B. Priority setting for health technology assessments: a systematic review of current practical approaches. Int J Technol Assess Health Care. 2007;23(3):310-5.

Phillips LD, Costa CAB. Transparent prioritisation, budgeting and resource allocation with multi-criteria decision analysis and decision conferencing. Ann Oper Res. 2007;154(1):51-68.

Prado CCL. A Adoção do limite custo-efetividade na incorporação de tecnologias no SUS - O que se pode esperar. Gestão e Saúde. 2015;6(Supl 4):3127-49.

Ritrovato M, Faggiano FC, Tedesco G, Derrico P. Decision-Oriented Health Technology Assessment: One Step Forward in Supporting the DecisionMaking Process in Hospitals. Value Health. 2015;18(4):505-11.

Saarni SI, Hofmann B, Lampe K, Lühmann D, Mäkelä M, Velasco-Garrido M, et al. Ethical analysis to improve decision-making on health technologies. Bull World Health Organ. 2008;86(8):617-23.

Shin T, Kim CB, Ahn YH, Kim HY, Cha BH, Uh Y, et al. The comparative evaluation of expanded national immunization policies in Korea using an analytic hierarchy process. Vaccine. 2009;27(5):792-802.

Simoens S. Health technologies for rare diseases: does conventional HTA still apply? Expert Rev Pharmacoecon Outcomes Res. 2014;14(3):315-7.

Sloane EB, Liberatore MJ, Nydick RL, Luo W, Chung Q. Using the analytic hierarchy process as a clinical engineering tool to facilitate an iterative, multidisciplinary, microeconomic health technology assessment. Comput Oper Res. 2003;30(10):1447-65.

Tannahill A. Beyond evidence - to ethics: a decision-making framework for health promotion, public health and health improvement. Health Promot Int. 2008;23(4):380-90.

Tervonen T, Naci H, van Valkenhoef G, Ades AE, Angelis A, Hillege HL, et al. Applying Multiple Criteria Decision Analysis to Comparative Benefit-Risk Assessment: Choosing among Statins in Primary Prevention. Med Decis Making. 2015;35(7):859-71.

Thokala P, Devlin N, Marsh K, Baltussen R, Boysen M, Kalo Z, et al. Multiple Criteria Decision Analysis for Health Care Decision Making - An Introduction: Report 1 of the ISPOR MCDA Emerging Good Practices Task Force. Value Health. 2016;19(1):1-13.

Thokala P, Duenas A. Multiple criteria decision analysis for health technology assessment. Value Health. 2012;15(8):1172-81.

Tony M, Wagner M, Khoury H, Rindress D, Papastavros T, Oh P, et al. Bridging health technology assessment (HTA) with multicriteria decision analyses (MCDA): field testing of the EVIDEM framework for coverage decisions by a public payer in Canada. BMC Health Serv Res. 2011;11:329.
Tromp N, Baltussen R. Mapping of multiple criteria for priority setting of health interventions: an aid for decision makers. BMC Health Serv Res. 2012;12:454.

University of York. NHS Centre for Reviews \& Dissemination. Providing reliable evidence to support decision-making: The NHS Economic Evaluation Database (NHS EED). University of York, NHS Centre for Reviews \& Dissemination; 2002.

Venhorst K, Zelle SG, Tromp N, Lauer JA. Multi-criteria decision analysis of breast cancer control in low- and middle- income countries: development of a rating tool for policy makers. Cost Eff Resour Alloc. 2014;12:13.

Vuorenkoski L, Toiviainen H, Hemminki E. Decision-making in priority setting for medicines - a review of empirical studies. Health Policy. 2008;86(1):1-9.

Wahlster P, Goetghebeur M, Kriza C, Niederländer C, Kolominsky-Rabas P; National Leading-Edge Cluster Medical Technologies 'Medical Valley EMN'. Balancing costs and benefits at different stages of medical innovation: a systematic review of Multi-criteria decision analysis (MCDA). BMC Health Serv Res. 2015;15:262.

Walton NA, Martin DK, Peter EH, Pringle DM, Singer PA. Priority setting and cardiac surgery: a qualitative case study. Health Policy. 2007;80(3):444-58.

Wilson EC, Rees J, Fordham RJ. Developing a prioritisation framework in an English Primary Care Trust. Cost Eff Resour Alloc. 2006;4:3.

Wilson E, Sussex J, Macleod C, Fordham R. Prioritizing health technologies in a Primary Care Trust. J Health Serv Res Policy. 2007;12(2):80-5.

Wilson KA, Carwardine J, Possingham HP. Setting conservation priorities. Ann N Y Acad Sci. 2009;1162:237-64.

Wirtz V, Cribb A, Barber N. Reimbursement decisions in health policy extending our understanding of the elements of decision-making. Health Policy. 2005;73(3):330-8.

Youngkong S. Application of HTA research on policy decision-making. J Med Assoc Thai. 2014;97 Suppl 5:S119-26.

Youngkong S, Baltussen R, Tantivess S, Mohara A, Teerawattananon Y. Multicriteria decision analysis for including health interventions in the universal health coverage benefit package in Thailand. Value Health. 2012a;15(6):961-70.

Youngkong S, Kapiriri L, Baltussen R. Setting priorities for health interventions in developing countries: a review of empirical studies. Trop Med Int Health. 2009;14(8):930-9.

Youngkong S, Teerawattananon Y, Tantivess S, Baltussen R. Multi-criteria decision analysis for setting priorities on HIV/AIDS interventions in Thailand. Health Res Policy Syst. 2012b;10:6. 